\title{
Reduced heart exposure of diclofenac by its polymeric micellar formulation, normalizes CYP- mediated metabolism of arachidonic acid imbalance in adjuvant arthritis rat model: Implications in reduced cardiovascular side effects of diclofenac by nano-drug delivery
}

\section{Hanan Al-Lawati, Mohammad Reza Vakili, Afsaneh Lavasanifar*, Surur Ahmed, Fakhreddin Jamali $^{*}$}

Faculty of Pharmacy and Pharmaceutical Sciences, University of Alberta, Edmonton, AB, Canada

\section{Supporting Information}
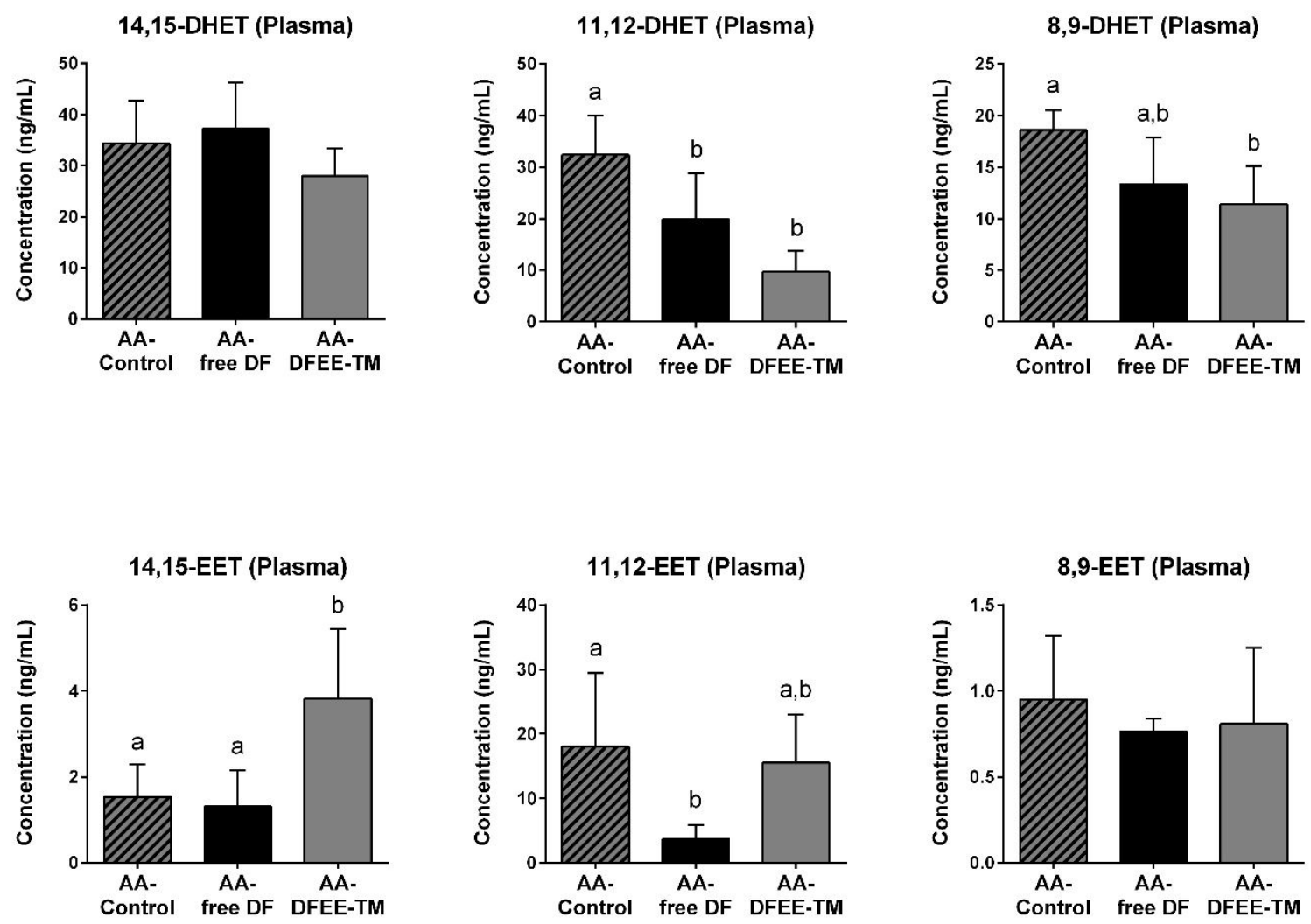

Figure S1. Concentrations of CYP metabolites of arachidonic acid (14,15-, 11,12- and 8,9-DHET, 14,15-, 11,12-, and 8,9-EET) in the plasma of adjuvant arthritis inflamed rats receiving no treatment (AA-Control), or treated with free diclofenac (AA-free DF), or with the diclofenac ethyl ester traceable micelles (AA-DFEE-TM) (n=6/group). Bars sharing the same letter are not significantly different based on one-way ANOVA $(\alpha=0.05)$ with post-hoc Tukey HSD. 

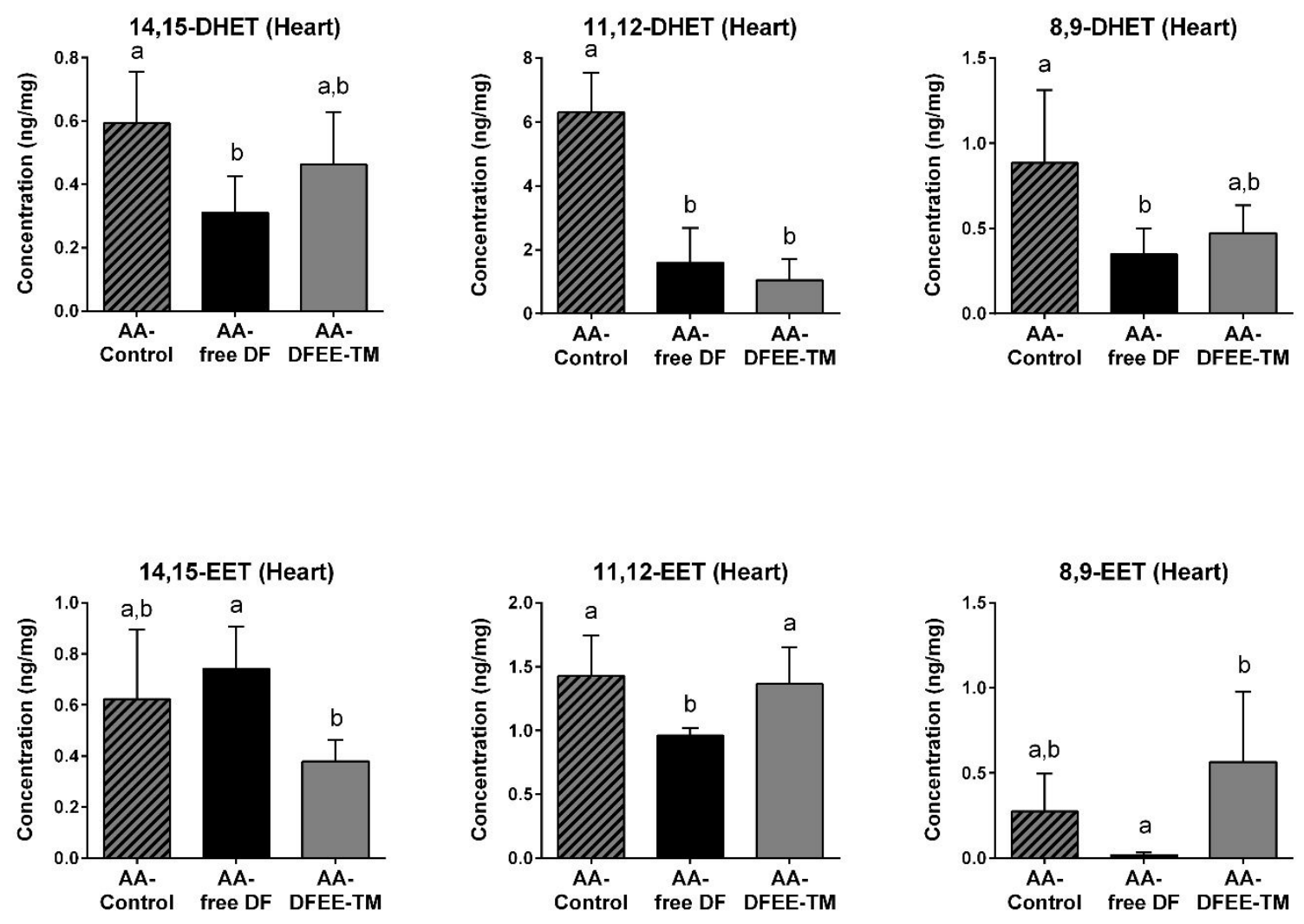

Figure S2. Concentrations of CYP metabolites of arachidonic acid (14,15-, 11,12- and 8,9-DHET, 14,15-, 11,12-, and 8,9-EET) in the cardiac tissues of adjuvant arthritis inflamed rats receiving no treatment (AA-Control), or treated with free diclofenac (AA-free DF), or with the diclofenac ethyl ester traceable micelles (AA-DFEE-TM) (n=6/group). Bars sharing the same letter are not significantly different based on one-way ANOVA $(\alpha=0.05)$ with post-hoc Tukey HSD. 

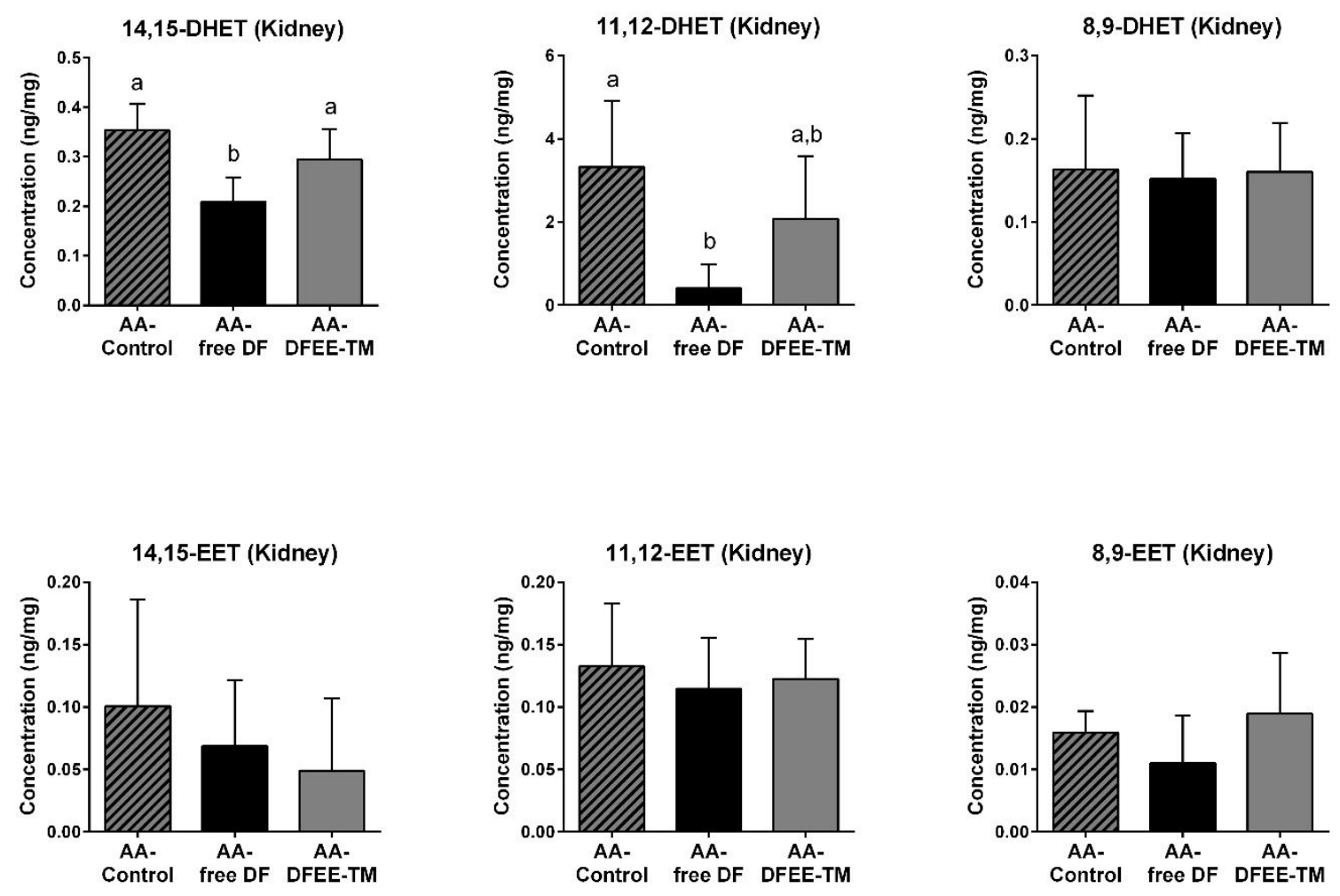

Figure S3. Concentrations of CYP metabolites of arachidonic acid (14,15- , 11,12- and 8,9-DHET, 14,15-, 11,12-, and 8,9-EET) in the kidney tissues of adjuvant arthritis inflamed rats receiving no treatment (AA-Control), or treated with free diclofenac (AA-free DF), or with the diclofenac ethyl ester traceable micelles (AA-DFEE-TM) ( $\mathrm{n}=6 /$ group). Bars sharing the same letter are not significantly different based on one-way ANOVA $(\alpha=0.05)$ with post-hoc Tukey HSD.
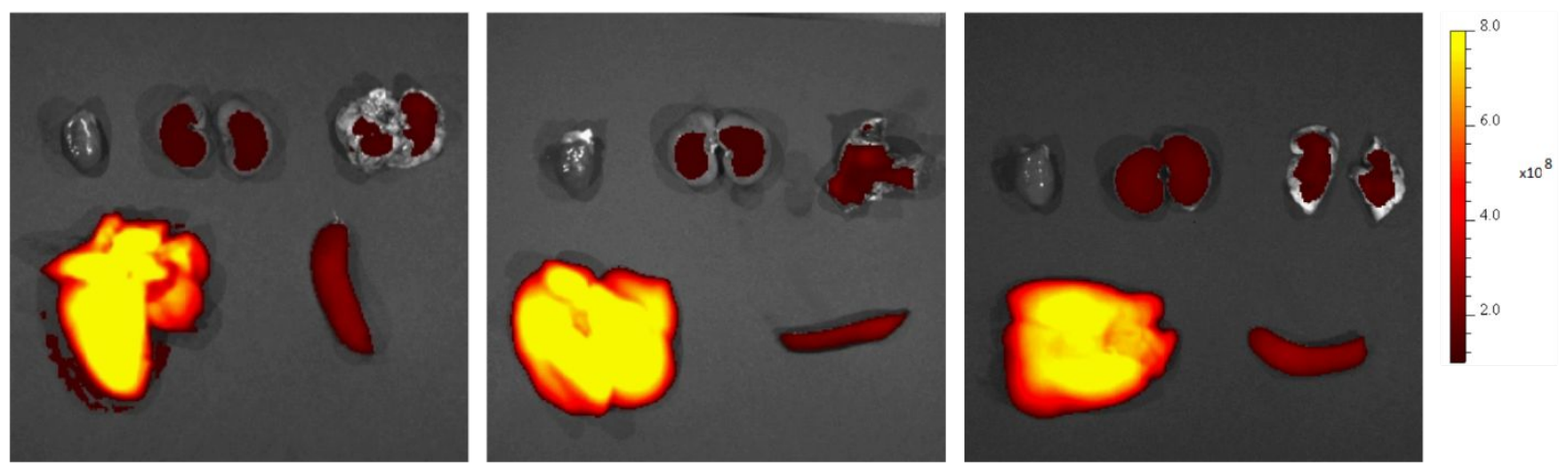
Figure S4. ex vivo near-infrared optical images of major organs (heart, kidneys, lungs, spleen, and liver in clockwise ordering) of adjuvant arthritic rats $(n=3)$ at $6 \mathrm{~h}$ following a single iv dose of the DFEE-TM. 